\title{
New Spin Isomers of Water Molecule
}

\author{
V.K.Konyukhov
}

A. M. Prokhorov General Physics Institute, Russian Academy of Sciences, Vavilov Street 38, 119991 Moscow, Russia

Copyright (c)2015 Horizon Research Publishing All rights reserved.

\begin{abstract}
It is proposed the new model of interplay of water molecule with solid surface, where molecules get new physical properties the result of which one can see, for example, in the fall of vapor pressure of $1 / 3$ compared to its original value. The water molecule loses two of three translation degrees of freedom, it loses also the full rotational spectrum but obtains mixed spin states instead of the pure original spin state which is provided by two protons inside the molecule. By taking into account the significant change in properties of molecules one can assert that the new spin isomers may exist under the certain environmental conditions. The predictions of the model are compared with experimental data within two adsorption models, one of which corresponds to the conventional case which suggests of keeping molecules on surface, and another case where molecules are found in gas. Two forms of the interpretation of experimental data give good results. The mathematical constructions of the Hopf map and of the geometric algebras are used during the creation of molecular models.
\end{abstract}

Keywords Ortho-water, Para-water, Adsorption on Solid Surfaces, Hopf Map

\section{Introduction}

There are several aspects of investigation of physical properties of water molecules, one of which consists in studying of a fully isolated molecule, or a molecule itself, by traditional methods of classical and quantum mechanics. Another aspect of investigation assumes, to the contrary, that the molecule interacts with classical or quantum bodies or more generally with its environment. It turns out, that simple quantum models for different degrees of freedom of the water molecule which have been developed today are not suitable to describe a relatively simple physical situation as a system of water vapor interacting with solid surface [13], [4], [14]. Additional complexities of the water-surface system comes to light when one considers a non-equilibrium state of spin isomers of water molecule [6].

A problem of existence for new forms of water molecule that will be investigated in this work is associated with the obligatory molecule-surface interaction and the finite lifetime of this kind of water molecule. A new powerful experimental method was introduced in the study of unresolved as yet problem of physical adsorption of water molecules on solid surfaces, which were elaborated in works devoted to non-equilibrium of spin isomers. This spectroscopic tool is very fast and precise for getting the relative concentration of water spin isomers in vapor and recalculating of measured values into the part of total gas pressure, provided by water molecules [15]. Thus we obtain two mutually independent means, namely the total gas pressure in vessel and the concentration of molecules in vapor to analyze in detail the state of water molecules.

The new form of existence or new kinds of state of water molecule have been indicated in [8],[9] and demands the appropriate mathematical methods [7] to describe old experimental observations. For the purposes of the following discussion it is convenient to denote the new form of water molecule as latent by taking into account their hidden behavior.

The main theoretical facility to be used is the notion of degrees of freedom (DF) of water molecule. We suppose that this concept is well defined for molecules with simple atomic structure, disregard of the classical or quantum description to be employed. In this work we will deal with three translational degrees of freedom (TDF) treated classically, with three rotational degrees of freedom (RDF) since the water molecule is an asymmetric quantum rotor, and with spin degrees of freedom (SDF), the number of which is defined by the number of spin states involved in consideration.

The physical properties of latent water molecules substantially differ from properties of ordinary molecules. They have only one TDF instead of three TDF in ordinary case. Pure rotational lines are absent in the infrared or submillimeter spectrum range instead of the infinite sequence of rotational lines in broad frequency range for ordinary water molecules. Spin states of latent molecules are as a rule mixed instead of ordinary case where states are commonly supposed to be pure.

At this place it is necessary to remind how is determined the adsorption ability of given substance. There is the vacuum chamber or vessel with a solid adsorbent inside which has been evacuated to a pressure considerably lower than a working pressure to be used. Then the vessel is filling by water vapor and an initial pressure $p_{i}$ is fixed. After a time when the pressure in vacuum chamber becomes constant and a dynamic equilibrium between adsorbed water molecules and those which was still contained in vapor, is achieved the total gas pressure $p$ also is fixed. It is suppose also that a leakage of the whole of vacuum system is sufficiently low to not distort the $p$ value. A difference of pressure $p_{i}-p$ is 
commonly regarded as a part of water molecules which was adsorbed on the surface of given adsorbent. The procedure described above is repeated again with other initial pressure of filling vapor so we obtain experimental points on an adsorption isotherm.

An approach to the adsorption problem which is associated with latent water molecules gives a new understanding of the pressure lowering effect usually observed in the adsorption experiments. An appearance of considerable amount of latent molecules in the vacuum vessel with one TDF leads to a reduction of the average number of collisions with walls which provides the gas pressure. The predicted value of latent molecules is the half of total number of water molecules in vessel. This value is achieved at the dynamic equilibrium between ordinary and latent molecules at the final time point of experiment.

The existence of latent molecules is obliged to an event of the physical adsorption of molecules on surface. Once the molecule is adsorbed, it loses three TDF in physical space and obtains instead of two TDF on a plane associated with surface. In course of desorption process the molecule may does not obtain again three TDF in physical space because it needs only one TDF to leave the surface. Informations about parameters of the plane may be stored on an ancillary or latent space an existence of which one can explain by following way.

Besides the physical 3D-space, there is another or latent 3D-space with properties which are similar to attributes of physical space if the molecule considered is itself the quantum rotor. The latent space belongs to the sphere $S^{3}$ with quaternions $\mathbf{i}, \mathbf{j}, \mathbf{k}$ as basis vectors and with real coordinates $x_{1}, x_{2}, x_{3}$ on $S^{3}$. Under certain conditions which may be fulfilled in experiments, the latent space is appeared in a description of translation motions of molecule, which results in the conclusion that the physical and latent spaces share the TDF. A division of the shared TDF of two for the latent space and of one for the physical space becomes quite obvious if we take into account the geometrical properties of spaces. The mathematical model of the physical space includes the point at infinity therefore it allows the movement of particles in one-dimensional subspace or simply on straight line without any restrictions in values of space coordinates. While the latent space, to the contrary, has the rigid constraint imposed on space coordinates that $x_{1}^{2}+x_{2}^{2}+x_{3}^{2} \leq 1$ which supposes the movement on planes with the finite values of space coordinates, for example on circle. Any movements in the latent space are at present unobservable by physical means.

The model of latent molecule with only one TDF and therefore it provides just of $1 / 3$ of gas pressure compared to usual water molecules, allows to get a quantitative description of the experiments with adsorption of water vapor on corundum ceramic that have been performed in [8].

\section{The experiment}

\subsection{Methodical part}

In almost all works devoted to the adsorption of water vapor on solid surfaces, relative variables such as vapor pressure or concentration of molecules in gas are ordinarily used to describe the adsorption process. The main problem by using relative variables consists in how to choose of sample units relative to which the variables were defined. So called the saturation vapor pressure is a typical example of such physical unit by means of which an isotherm of adsorption is created. Another problem arises if several relative variables are used, then the following method can be applied.

If we intend to compare two collections of experimental data, namely results of measuring of the integral intensity of spectral lines for para and ortho-molecules in our case, one must reduce to a common scale the experimental points for given two collections. To make this task we must choose a point with maximum value for every of collections independently and then divide all points of given collection by using the maximum-valued points. So all already relative points of the collections will fall within a scale range $[0,1]$. We expect that experimental points of collection in our case will not exceed an initial point therefore the normalization process was carried out for ortho-molecules or para-molecules or pressure in vacuum chamber by using their initial points. The result of such treating of experimental data was placed in Figure 1.

The manner of rescaling and the introduction of relative variables have a negative property, as we lose all information about any parameters of spectral lines, that can be represented as numerical coefficient or multiplication factor in measured results. In particular, the nuclear statistical weights of isomers at initial moment of experiment have been erased.

The nuclear statistical weight is a numerical characteristic of quantum system which obtains the water molecule because it has the SDF that arises due to nuclear spin moments of protons. This important quantum parameter one must use if we deal with the electronic or vibrational or rotational DF of molecule and if we need to divide they into parapart and ortho-part. This parameter describes how the water molecules shared of four spin states provided by nuclear spin of protons. It is suggested as usual that every spin state is associated on average with equal number of molecules, hence in other words, only one molecule corresponds to every spin state. There is the most known spin phase with four pure states which holds one para-state and three ortho-states denoted as $\alpha$ phase (B2). This is the case where each onedimensional subspace of the Hilbert space holds the one spin state.

In more general cases we have two-dimensional or threedimensional subspaces in the Hilbert space which holds accordingly two or three spin states. We make the significant assumption that only one molecule corresponds again to every many-dimensional subspace where states involved generate the mixed spin state with statistical weights equal to the dimension of subspaces, denoted farther as $\beta$ and $\gamma$ spin phases (B2). Note that all reasoning about many dimensional subspaces are right being applicable to the ortho-side of the Hilbert space because the para-side is always one dimensional with one pure spin state.

The nuclear weight is needed to calculate a relative pressure in vacuum chamber by using relative dimensionless variables $v_{o}, v_{p}$ that are presented in Figure 1. One can restore the nuclear statistical weights with weighted constants $K_{o}, K_{p}$. All possible variants of spin composition of water vapor at initial moment are demonstrated in (1). It is important to note that the calculated relative pressure $p_{f}$ is produced just by a part of those water molecules which we can detect by the spectroscopic method employed. In order to emphasize this fact we add to the pressure symbol an index $f$ that will denote free or ordinary molecules which were at initial moment in vacuum chamber. 


\begin{tabular}{|c|c|c|}
\hline \multicolumn{3}{|c|}{$p_{f}=K_{o} v_{o}+K_{p} v_{p}$} \\
\hline & $K_{o}$ & $K_{p}$ \\
\hline & $1 / 2$ & $1 / 2$ \\
\hline & $2 / 3$ & $1 / 3$ \\
\hline & $3 / 4$ & $1 / 4$ \\
\hline
\end{tabular}

It is worth to note, that the equation on the first line in (1) does not depend on the number of water molecules in unit volume of vapor, not only because the relative variables used but also because we employed an optical thin layer by measuring the amount of adsorption within spectral lines.

A peculiar effect for ordinary water molecules associated with r-adsorption may be observed if molecules change their spin-phase under condition of fixed number of molecules in vessel. The prefix $r$ - is added to distinguish an adsorption for the resonance spectral radiation from the adsorption on surface.

Assume as a basis for our reasonings the established physical fact that r-adsorption is proportional to the number of molecules in unit volume of gas. Suppose also that the equal number of molecules on average are in each spin subspaces of the Hilbert spaces of water molecule disregarding the dimension of subspaces, or in other words, paying no attention to the result of pure or mixed spin states are found in the subspaces. The last supposing is right because the energy associated with SDF is much less than thermal energy of gas. If we make an additional assumption that the general amount of water molecules in vessel is fixed then the change of spin phase for ordinary water molecules yields different values of the r-adsorptions.

An explanation of this effect is based on the different number of subspaces for each spin-phase which are permissible to be occupied by molecules and a redistribution of molecules between subspaces. The $\alpha$-phase has 4 such subspaces, the $\beta$-phase has 3 but the $\gamma$-phase has just 2 . The result of change of r-adsorption can be calculated by formula to link, for example, the $\alpha$-phase and $\beta$-phase.

$$
\begin{aligned}
& r=\frac{w_{\beta}}{w_{\alpha}+w_{\beta}} v_{\alpha}+\frac{w_{\alpha}}{w_{\alpha}+w_{\beta}} v_{\beta} \\
& v_{\alpha}+v_{\beta}=1 \quad w_{\alpha}=4 \quad w_{\beta}=3
\end{aligned}
$$

If the gas of ordinary water molecules is originally in $\alpha$ phase the conversion into $\beta$-phase or $\gamma$-phase yields the increase of r-adsorption of $4 / 3$ or 2 times respectively.

\subsection{Experimental part}

An experimental installation to study the adsorption of water vapor on solid surface can be presented by following way [8]. Originally our experimental setup was constructed to observe the isomeric non-equilibrium of water vapor but now we can think of this device as a convenient instrument to obtain several points on the adsorption isotherm.

Before beginning the description of experimental conditions which have been used in this article one needs to remember how to prepare solid surfaces to be active in the isomeric non-equilibrium experiments. In contrast to world trend in preparation of solid surfaces which includes the high vacuum equipments and cleanliness of surfaces, the practise of surface preparation for non-equilibrium observation demands that surfaces must be covered by certain amount of water molecules which form centers of adsorption. These centers play the crucial role in creating the isomeric nonequilibrium effect. If adsorption surfaces are fully dry then any effect of non-equilibrium can not be observed [10]. The water molecules on surface provide a ground or threshold gas pressure in vacuum chamber which is evaluated for circumstance of our experiment ca 50 mTorr, hence the high vacuum technique is not applicable here.

There is a vacuum chamber with walls that was made from stainless steel or teflon in order to have a sufficiently large relaxation time into isomeric equilibrium state. The solid adsorbent in the form of corundum ceramic balls of $2 \mathrm{~cm}$ in diameter are placed into the chamber. The surface of balls was not purified by any technique hence some atmospheric molecules was adsorbed on surface. Next the chamber is evacuated to the pressure about $10^{-2}$ Torr at room temperature and then the vessel is filled by water vapor at pressure range from 3 to 5 Torr. There is the measuring system that records the population of rotational levels of free molecules and pressure in chamber as functions of time.

We assume that the population of vibrational-rotational states of free molecules are at the equilibrium and the spin isomers of free molecules behave as different gases due to the strong quantum prohibition for transitions between isomers. From experiments that were carried out on this setup one can see that the measured values of population of molecular levels and pressure in chamber become stationary 2040 minutes later the moment of injection of water vapor into chamber, see Figure 1. According to the ordinarily or our previous interpretation of experiments the pressure decrease and the concentration fall of para-isomer and ortho-isomer indicate that an adsorption on surface of ceramic balls takes place.

According to the statistical treating of the four last experimental points on ortho-curve or para-curve or pressure-curve we have following values of $v_{o}=0.731 \pm 0.012, v_{p}=0.431 \pm$ $0.013, p_{f}=0.658 \pm 0.003$ which appropriate to stationary conditions. Comparing the calculated values $p_{f}$ for different spin phases of water molecule according to (1) we lead to conclusion that the water vapor occupies $\alpha$-phase, and calculated value $p_{f}=0.656$ coincides with experimental $p_{f}$ within an error. Hence the ordinary point of view on the adsorption process with keeping water molecules on surface get an positive confirmation.

There is another possible interpretation of the experiments with water molecules and the ceramic balls, the solid surface of which plays a role of catalyst to convert ordinary water molecules into latent molecules. This model of interaction of molecules with surface does not suppose that a considerable amount of molecules is kept on surface. A short time adsorptions are permitted, during which the conversion from ordinary state into latent state and vice versa for molecules occur, hence almost all molecules both ordinary and latent are found in gas phase. It turns out that there are the half of ordinary molecules and the half of latent molecules in vacuum vessel because the gas-phase reaction takes place between ordinary and latent molecules. The reaction has no energy barrier, hence the reaction rate is equal in both directions and if an dynamic equilibrium is reached then the concentrations of initial substance and reaction products must be equal.

For the purposes of the following discussion it is convenient to use simple fractions for relative values of concentrations and pressures involved in consideration. We associate the final pressure in vacuum vessel with $2 / 3$ since the exper- 
imental value is very near to this fraction and the number of ordinary or latent molecules in unit volume or in the whole of vacuum vessel with $1 / 2$ at final time point of process.

According to the ideal gas law we can write the proportion between the total pressure $p$ and number of molecules of two kinds which are found in vessel by omitting the general proportional factor. The relative variables $v_{3}, v_{1}$ are appropriated to parts for each kinds of molecules and subscripts indicate the number of their TDF.

$$
p=v_{3}+\frac{1}{3} v_{1} \quad v_{3}+v_{1}=1
$$

The first term is related with ordinary water molecules but the second term belongs to latent molecules with factor $1 / 3$ which takes into account that latent molecules have only one active TDF instead of three TDF in the case of ordinary molecules. So we can explain the final total pressure $2 / 3$ and also the pressure fall of $1 / 3$ as the result of loss of two TDF for latent molecules. The result is that ordinary and latent molecules together provide gas pressure which coincides with measured value, hence the model of latent molecules provides the right description of pressure behavior.

The following step of new approach to the problem of interaction of water molecules with ceramic balls is a calculation of the pressure value in vacuum chamber based on $r$ adsorption data which are provided by ordinary molecules.

So far we considered the collection of ordinary water molecules as a part of general amount of molecules which remain unchanged during production of latent molecules. This assumption is not right since the ordinary molecules change their spin phase from initial $\alpha$-phase to $\beta$-phase which results in an increase of $\mathrm{r}$-adsorption for both para-molecules and ortho-molecules. This result is based on the following reason.

According to the basic principles of quantum mechanics, if two quantum systems interact with each other during certain time and therefore they become entangled, then after the time moment when the systems are disjointed they must be itself in mixed quantum states. This rule can be applied to spin system of water molecule as the first quantum system and to spin system of other water molecule kept on surface as the second system. An interplay time is the time of adsorption and the mixed spin state can arise on the ortho-side of spin system for molecule which comes back into gas, all three states of which was originally in the pure states before adsorption. We can conclude that ordinary water molecules must occupy the $\beta$-phase after a time, provided the short time interaction of molecules with surfaces of ceramic balls takes place.

By choosing the unit of number for ordinary water molecules we suppose that unit for r-adsorption is related to the number unit in the proper way so that a relative variable $v_{r}$ for r-adsorption and a relative variable $v_{n}$ for number are equal to 1 at initial moment of experiment. At final moment of process when $v_{n}=1 / 2$ and a comparison between calculated and measured values of pressure is made the value of $v_{r}$ can be presented by formula

$$
v_{r}=v_{n} \frac{4}{3}=\frac{2}{3}
$$

where increase factor $4 / 3$ was taken from (2)

The result is that the model of adsorption of water molecules using latent molecules well describe the vapor pressure in vacuum chamber.

\section{Discussion}

There are several topics to discuss in this Section which we divide into Subsections to organize the process, however all topics are associated with each other in many cases.

\subsection{Spin part}

To date there are a great number of publications devoted to quantum system of two half-spin particles involved. The system has been considered with different points of view and the content of publications has a different degree of importance. A purpose of our consideration of this quantum system is how to apply mathematical constructions used in this area to describe in detail the SDF for water molecule.

First, we change the accepted sequence of basis vectors of the Hilbert space known as calculating basis to the form $\{|10\rangle,|01\rangle,|11\rangle,|00\rangle\}$ denoted further as c-basis. Second, we represent all matrices that are needed to describe spin states, density matrices, projection operators, as a direct sum of two-dimension matrices, because four-dimensional matrices that will be used in our considerations, have no offdiagonal elements that are capable of destroying the direct sum constructions (B1). For example, an operator which changes the sequence of the first two basic vectors has the form

$$
\left\|\begin{array}{ll}
0 & 1 \\
1 & 0
\end{array}\right\| \bigoplus\left\|\begin{array}{ll}
1 & 0 \\
0 & 1
\end{array}\right\|
$$

However, we introduce another coordinate system in the Hilbert space by making superpositions from the first two basis vectors $\{|10\rangle-|01\rangle,|10\rangle+|01\rangle,|11\rangle,|00\rangle\}$ denoted further as s-basis. Usually this change of coordinate system is related with a transition to the basis of irreducible representations by selecting 1D and 3D subspaces [17]. We have another view on this transformation, we can think of the s-basis as a manner of introducing of symmetry properties in spin system. Really, if we apply the symmetry operator (5) to vectors of c-basis arranged as a column, the first vector of s-basis changes its sign while other three vectors remain unchanged under an essential condition that the transition matrix which maps the c-basis onto the s-basis is also unchanged. This observation makes a symmetry classification of spin states for water molecule.

The division of the Hilbert space into the antisymmetrical 1D or para subspace and the symmetrical 3D or otho subspace plays a central role by constructing spin isomers of water molecule. One can obtain a singlet wave function from the first basis vector and all three triplet wave functions using other basis vectors. One can introduce the nuclear statistical weights for singlet and triplet states as to be equal to the number of subspaces by using further its weighted form $1 / 4$, $3 / 4$.

The following portion of our consideration is devoted just to the ortho subspace or ortho-side with the aim to study properties of all possible subspaces of the ortho-side. There is three ways how to choose subspaces denoted by Greek letters: $\alpha$ [three 1D], $\beta$ [one 1D, one 2D], $\gamma$ [one 3D]. All three variants have the physical sense and particular properties by considering their as separate spin phases $(\mathrm{B} 1, \mathrm{~B} 2)$.

An essential assumption was made about dimensions of spin subspace which can be associated with a distinct water molecule. Now it is supposed as usual, that the molecule can 
hold only an one-dimensional spin subspace with the pure wave function so we can use just the $\alpha$ phase among of available spin states. An extension of accessible spin states by $2 \mathrm{D}$ and 3D subspaces supposes that 2D and 3D spin states are mixed quantum states with equal weights $1 / 2$ or $1 / 3$ of pure states involved. An idea about mixed spin states in experiments with spin isomers of water molecules has been published in [6].

Now there exists a point of view on capability of solid surfaces to produce the ortho-para non-equilibrium in water vapor which divides all solid matters into active and non-active depending on the appearance of non-equilibrium in experimental test. Usually, it is observed the magnification of amount of the ortho-isomer in gas phase [6], [7]. But there is only one experiment with the liquid glycerol where an enrichment by para-isomer has been observed [16].

There are two channels for ortho-molecules and one channel for para-molecules that transmit free molecules into latent molecules and vice versa with equal rates [7]. If the number ortho-channels coincides with the number of ortho spin states as in the case of $\beta$ phase, the non-equilibrium of free molecules is absent, but the conversion process itself takes place. It is not hard to show that the non-equilibrium shifts into ortho-side in the $\alpha$ phase and into the pata-side for $\gamma$ phase because in $\alpha$ case is the lack of one additional channel, but in $\gamma$ case there is one redundant channel. The side and magnitude of these deviations depend above all on a liquid water sample which has been chosen. One can test many samples of water and surfaces by observing the vapor pressure and comparing its value with predicted value of $2 / 3$.

\subsection{Rotational part}

The rotational degrees of freedom need to be sufficiently in detail commented not only by itself but also due to their relation with TDF of water molecule. A description of quantum rotational motion of polyatomic molecules has an origin based on matrix representations of group SU(2). The infinite sequence of representations by unitary hence complexvalued matrices with integral weights or quantum numbers is the ground of all constructions known at present. Every matrix is regarded as an independent Hilbert space and other elements needed namely, matrices of angular momentum, the rotational Hamiltonian are built by using items of matrix as basis vectors of the Hilbert space. This process was described many times in textbooks or original articles with a different level of comprehension. Several references are in [7].

A main idea of the present study based on the representation of group $\mathrm{SU}(2)$ with the orthogonal matrix by realvalued items. Such matrix is unique and well-known, it is matrix $\mathrm{SO}(3)$. There are several ways to get matrix $\mathrm{SO}(3)$ which we will consider later, but here it is necessary to note some geometrical aspects of an issue under discussion.

The three-dimensional sphere $S^{3}$ where rotational wave functions are defined, holds the three-dimensional subspace that can be regarded as a particular model of the physical $3 \mathrm{D}$-space with the quaternions $\mathbf{i}, \mathbf{j}, \mathbf{k}$ as vectors of the coordinate system and with an important restriction on coordinates $x_{1}, x_{2}, x_{3}$ that $x_{1}^{2}+x_{2}^{2}+x_{3}^{2} \leq 1$. This "latent" model of $3 \mathrm{D}$-space can be used to describe an object that is moving on closed orbits while this model is not suitable for representing a rectilinear motion of particles. Thus it means that there is another 3D-space along with the latent space which has not the limiting conditions that was imposed on coordinates. Such conditions are fulfilled for the ordinary physical 3D-space. An existence of two local isomorphic 3D-space allows to describe classical or quantum systems with three degrees of freedom by using both spaces at the same time. A difference between properties of the latent and the physical $3 \mathrm{D}$-spaces one can see by using the stereographic projection of $S^{3}$ onto 3D-space. The projection can be performed if we use just the physical 3D-space because the last space holds the point at infinity but the first space has not.

We can think of coordinates $x_{1}, x_{2}, x_{3}$ on $S^{3}$ as the space coordinates of a point mass in 3D-space, so the important relation is built between RDF and TDF of molecules. Later this connection will be destroyed due to an introduction of complex variables to describe the quantum rotation of molecules. However, this essential connection arises again when one needs to describe the rotation of molecule by real variables.

From a geometrical point of view any rotation in 3D-space has an axis of rotation and an angle of rotation [2]. Every rotation axis can be represented by a vector $\mathbf{r}$ of unit length, so a collection of all such vectors forms the sphere $S^{2}$. To define the rotation angle, one needs a plane with a circle $S^{1}$ of unit radius that holds two points on circle, one of which is fixed and another is used to indicate the angle of rotation. The axis is normal to the plane and a direct line along axis intersects the plane in central point of circle.

Using this relatively simple geometrical construction we can explain how to work the Hopf map from $S^{3}$ onto $S^{2}$ that is a particular case of the fiber bundle. For every map it is interesting to know what a collection of elements of $S^{3}$ corresponds to a single point from $S^{2}$. It turns out that the collection of items is just all points of $S^{1}$ which is an object of the latent 3D-space, in other words, making the map we take into account only axes of rotation that belongs to the physical $3 \mathrm{D}$-space and disregard angles of rotation. It is important to understand that the vector $\mathbf{r}$ represents the one-dimensional subspace in physical 3D-space. Now we deal not with the physical 3D-space as a whole, but with the set of 1D-spaces.

As a rule, the fiber bundle exists not in a distinct version, there are several kinds of the bundle that appropriate to additional geometric factors. In our case, one can obtain three fully equivalent fiber bundle by choosing one of three possible $\mathbf{i}, \mathbf{j}, \mathbf{k}$ quaternions as an ancillary object, so we get three sets of coordinates associated with three rows of the matrix $\mathrm{SO}(3)$ for the same vector $\mathbf{r}$, see (A10). Comparing the rows of matrix $\mathrm{SO}(3)$, one can see that four coordinates $x_{0}, x_{1}, x_{2}, x_{3}$ on sphere $S^{3}$ appear in expressions of matrix elements by an equivalent manner, and the coordinates don't hold any information how the reference system has been chosen in the physical 3D-space.

If we assume that the translation motion of molecules needs to describe by the same variables $x_{1}, x_{2}, x_{3}$ as the rotational motion, then a displacing two of three TDF from the physical 3D-space into the latent 3D-space becomes inevitable. Two variables involved in the latent 3D space provide description of an orbital motion of molecule about center of attraction, if it is necessary. Vectors for rotation $\mathbf{r}_{\mathbf{r}}$ and vector for translation $\mathbf{r}_{\mathbf{t}}$ of a distinct molecule sometimes can coincide.

The one-dimensional translational motion of molecule is very convenient to describe a gas pressure from the kinetic point of view because molecules before and after collisions take place with walls move along straight lines and more 
complicated paths are excluded. The gas which contains just such molecules, provides three times less of the total gas pressure compared to gas of particles with three TDF.

\subsection{Experimental part}

The result of experimental work described in given article can be regarded as a new type of interplay between water vapor and solid surface that leads to the pressure fall which are usually observed in almost all adsorption experiments. But this decrease in pressure is not the result of keeping of molecules on surface according to conventional point of view. The interaction of molecules with the ceramic surface covered partly by adsorbed water molecules change the properties of gas molecule in such way so that the half of amount of gas molecules lose two own TDF that yields the pressure decrease.

We have shown in Seq. 2 how the model with latent molecules can explain the value of $2 / 3$ for the vapor pressure in the vacuum chamber measured by diaphragm instrument. Here we will show how this model explains also the value of the concentration of ordinary water molecules based on the experimental values $v_{o}, v_{p}$ of r-adsorption for ordinary water molecules at final moment of experiment.

The concentration $c_{\beta}$ of ordinary water molecules can be presented by following formula, where the index $\beta$ indicates that molecules are found in the $\beta$-phase.

$$
c_{\beta}=\left(3 v_{o} / 4+v_{p} / 4\right) \frac{3}{4}=0.49 \pm 0.02
$$

We regard the measured values $v_{o}=0.73 \pm 0.01$, $v_{p}=0.43 \pm 0.01$ as parameters of the $\alpha$-phase and multiply their sum by transition factor taken from (2) to convert the calculated value into another value appropriate to the $\beta$-phase. One can see that the concentration $c_{\beta}$ equals to $1 / 2$ within an error to the general amount of water molecules in chamber, hence the model with latent molecules is suitable to employ also in r-adsorption case.
An appearance of the spin-phase of water molecules with mixed spin states, namely beta-phase or gamma-phase instead of the originally alpha-phase existed is an inevitable sequence of interplay of molecule SDF with other proton spin moments which belongs to molecules kept on surface [5].

\section{Conclusion}

In this work is described the experiment of adsorption of water vapor on solid surface in its classical arrangement, included as usual the gas pressure measurement. But the measurement tools is extended by the spectroscopic equipment which works at the submillimeter wave range and uses rotational spectral lines of water molecule, that allow to us to calculate the vapor pressure produced by water molecules at subsequent time moments, relative to the vapor pressure at initial moment of experiment.

Subsequent steps in performing the experiment was carried out in accordance with the conventional procedure adopted in adsorption studies. We observed the pressure fall of $1 / 3$ at final time point and the decrease of adsorption of resonance radiation on rotational lines for both para-molecules and orthomolecules at the same amount, which usually associated with adsorption of water molecules on surface. 


\section{Appendix A}

A purpose of this Appendix is to show how to go from the rotational wave functions of free or ordinary molecule to the rotational wave functions of latent state using a detailed description of successive steps. For convenience, we will use different notations for elements of representation matrices of the SU(2) group in accordance with their properties considered.

The matrix of SU(2) group can be written in two forms

$$
\left[\begin{array}{cc}
\alpha & \beta \\
\gamma & \delta
\end{array}\right]=\left[\begin{array}{cc}
x_{0}+i x_{1} & x_{2}+i x_{3} \\
-x_{2}+i x_{3} & x_{0}-i x_{1}
\end{array}\right]=x_{0} \mathbf{1}+x_{1} \mathbf{i}+x_{2} \mathbf{j}+x_{3} \mathbf{k}
$$

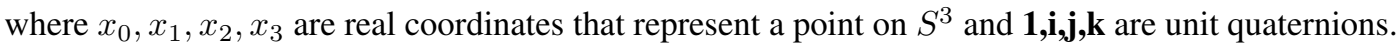

A representation matrix for the quantum number of angular momentum $\mathrm{L}=2$, was obtained by using the method described in [17] and based on a combination of elements of the first matrix listed above.

$$
\left.\begin{array}{ccccc}
\delta^{4} & 2 \gamma \delta^{3} & \sqrt{6} \gamma^{2} \delta^{2} & 2 \gamma^{3} \delta & \gamma^{4} \\
2 \delta^{3} \beta & \delta^{2}(\alpha \delta+3 \beta \gamma) & \sqrt{6} \delta \gamma(\alpha \delta+\beta \gamma) & \gamma^{2}(\beta \gamma+3 \alpha \delta) & 2 \gamma^{3} \alpha \\
\sqrt{6} \delta^{2} \beta^{2} & \sqrt{6} \beta \delta(\alpha \delta+\beta \gamma) & \left(\alpha^{2} \delta^{2}+4 \gamma \delta \alpha \beta+\gamma^{2} \beta^{2}\right) / 2 & \sqrt{6} \alpha \gamma(\alpha \delta+\beta \gamma) & \sqrt{6} \gamma^{2} \alpha^{2} \\
2 \delta \beta^{3} & \beta^{2}(\beta \gamma+3 \alpha \delta) & \sqrt{6} \alpha \beta(\alpha \delta+\beta \gamma) & \alpha^{2}(\alpha \delta+3 \beta \gamma) & 2 \gamma \alpha^{3} \\
\beta^{4} & 2 \alpha \beta^{3} & \sqrt{6} \alpha^{2} \beta^{2} & 2 \alpha^{3} \beta & \alpha^{4}
\end{array}\right]
$$

The elements of every matrix row has a symmetry with regard to interchange of variables that were involved, can be described by a substitution

$$
\left[\begin{array}{llll}
\alpha & \beta & \gamma & \delta \\
\beta & \alpha & \delta & \gamma
\end{array}\right]
$$

One can derive linear combinations of columns that have properties to be even or odd under an action of the substitution, so we obtain a group with elements $\{\mathrm{e}, \mathrm{o}\}$ that can be used for classification purpose.

$$
\left[\begin{array}{lllll}
a_{2,2}+a_{2,-2} & a_{2,0} & a_{2,1}+a_{2,-1} & a_{2,1}-a_{2,-1} & a_{2,2}-a_{2,-2} \\
a_{1,2}+a_{1,-2} & a_{1,0} & a_{1,1}+a_{1,-1} & a_{1,1}-a_{1,-1} & a_{1,2}-a_{1,-2} \\
a_{0,2}+a_{0,-2} & a_{0,0} & a_{0,1}+a_{0,-1} & a_{0,1}-a_{0,-1} & a_{0,2}-a_{0,-2} \\
a_{-1,2}+a_{-1,-2} & a_{-1,0} & a_{-1,1}+a_{-1,-1} & a_{-1,1}-a_{-1,-1} & a_{-1,2}-a_{-1,-2} \\
a_{-2,2}+a_{-2,-2} & a_{-2,0} & a_{-2,1}+a_{-2,-1} & a_{-2,1}-a_{-2,-1} & a_{-2,2}-a_{-2,-2} \\
A & A & B_{3} & B_{2} & B_{1}
\end{array}\right]
$$

It gives the first part of a symmetry classification of rotational wave function that is associated with a $D_{2}$ group [11] by representing the symmetry properties of the Hamiltonian of asymmetric rotor. The second part of the classification based on a parity of column indexes of the matrix elements is also the group of order 2 and the full $D_{2}$ group is presented as a direct product of two subgroups.

Representations of $D_{2}$ group denoted as $A, A_{1}, A_{2}, A_{3}$ are given by four numbers of 1 with a sign plus or a sign minus according to an amount of elements in the commutative $D_{2}$ group of order 4 . For purposes of the water molecule spectroscopy it is convenient to think of $D_{2}$ and its representations as being divided up into subgroup $\{$ ee,oo $\}$ and coset $\{$ eo,oe $\}$. This simple mathematical fact can be verified by using items of (A4), where we have gathered all the information about the elements of group $D_{2}$ and its representations.

$$
\left[\begin{array}{rrrrr}
\text { ee } & A & 1 & 1 & 1 \\
\text { оо } & B_{2} & -1 & -1 & 1 \\
\text { eо } & B_{3} & 1 & -1 & -1 \\
\text { ое } & B_{1} & -1 & 1 & -1
\end{array}\right]
$$

If we want to obtain, for example, the result for two elements that combine according to the group rule, then it is enough to multiply appropriate numbers in columns. The last column of (A4) provides the symmetry type of objects described by a proper representation.

The decomposition of $D_{2}$ group into the subgroup and its coset, has a great significance for spin isomers of water molecule. The para-isomer with symmetrical rotation wave function, in present notation even, and the ortho-isomer having antisymmetrical or odd rotational wave function are associated with the subgroup or the coset accordingly and they really are substantially different mathematical objects. The subgroup sets selection rules for quantum transitions between rotational levels in each spin isomer, but the coset give rise to transitions between isomers. A discussion about the possibility to observe the last type of transitions in water molecules one can find in [3], [12].

It is worth relating the classification of rotational states or wave functions of the asymmetric rotor with symmetry properties of the water molecule. While the symmetry classification group $C_{2 v}$ of the water molecule is isomorphic to the $D_{2}$ group, we 
use its subgroup of the order 2 involving a two-fold symmetry axis. This restriction can be explained by taking into account that we just use a plane figure of molecule which is formed by nuclei of molecule, disregarding an electronic cloud that makes the figure of molecule to be a three-dimensional shape. In spectroscopy the water molecule is known as b-molecule because its dipole moment is directed along the symmetry axis or Oy axis of the molecular reference system. This property gives rise to radiation transitions separately in the para-spectrum and in the otho-spectrum of molecules therefore the vector of dipole moment and $x_{2}$ variable have the type $B_{2}$ of classification. The other axes of the molecular reference system are an axis Ox variable $x_{1}$ and an axis $\mathrm{Oz}$ variable $x_{3}$ one can describe by type $B_{1}$ and $B_{3}$ accordingly. The symmetry classification based on the group of the plane molecular model does not contradict with the classification of representations of $D_{2}$ group using the notions of subgroup and coset. This fact shows that the classification based on the properties of asymmetric rotor is suitable for the water molecule.

In following discussions of energy properties of rotational states we restrict oneself to three last columns on (A4) because the elements of those columns are eigenstates of the rotational Hamiltonian of asymmetric rotor. If one employs variables $x_{0}, x_{1}, x_{2}, x_{3}$ on $S^{3}$ then Hermit differential operators representing angular momentum have the form

$$
\begin{aligned}
& L_{1}=i \hbar\left(x_{3} \frac{\partial}{\partial x_{2}}-x_{2} \frac{\partial}{\partial x_{3}}\right)+i \hbar\left(x_{0} \frac{\partial}{\partial x_{1}}-x_{1} \frac{\partial}{\partial x_{0}}\right) \\
& L_{2}=i \hbar\left(x_{1} \frac{\partial}{\partial x_{3}}-x_{3} \frac{\partial}{\partial x_{1}}\right)+i \hbar\left(x_{0} \frac{\partial}{\partial x_{2}}-x_{2} \frac{\partial}{\partial x_{0}}\right) \\
& L_{3}=i \hbar\left(x_{2} \frac{\partial}{\partial x_{1}}-x_{1} \frac{\partial}{\partial x_{2}}\right)+i \hbar\left(x_{0} \frac{\partial}{\partial x_{3}}-x_{3} \frac{\partial}{\partial x_{0}}\right)
\end{aligned}
$$

The rotational Hamiltonian has the rotational constants $c<b<a$ of the water molecule.

$$
\mathcal{H}=c L_{1}\left(L_{1}(\psi)\right)+b L_{2}\left(L_{2}(\psi)\right)+a L_{3}\left(L_{3}(\psi)\right)
$$

If we restrict oneself to one column then one can see that for every row the rotational wave function has various forms. Nevertheless, they have the same energy value and therefore the rotational level has a five-fold degeneracy. This fundamental property is typical for all kinds of quantum rotors. We have collected a required information about spectroscopic indication of rotational wave function, their type of symmetry and energy of rotational levels for water molecule in (A6).

$$
\left[\begin{array}{ccccc}
2_{21} & B_{3} & c+b+4 a & 135.3 & x_{1} x_{2} \\
2_{11} & B_{2} & c+4 b+a & 95.2 & x_{1} x_{3} \\
2_{12} & B_{1} & 4 c+b+a & 79.5 & x_{2} x_{3}
\end{array}\right]
$$

The first essential step in a direction which has been announced above is a notion of direct and reverse rotations as applied to rotation of molecules. The additional two-fold degeneracy of the rotation of molecules can be introduced in several ways.

To describe the motion of asymmetric rotor, one can use as basis functions of the Hilbert space the columns of representation matrix as well as its rows. Such an opportunity is provided by the unitary structure of matrix. Another approach to the problem of direct and reverse rotations rests upon the successive using of reverse representation matrix by obtaining results described above. One can show that the energy of rotation levels and the symmetry classification of wave function are identical to values for the previous case. There is one more method to get all objects such as matrices, operators and functions in the reverse rotation case. This manner is presented as the changing of signs before variables $x_{1}, x_{2}, x_{3}$ to opposite value. For example, the collection of angular momentum operators for reverse rotation one can obtain from (A5) by changing signs before three of four variables.

To take into account the reverse rotation one can employ the next manner. Since rotational levels of molecule have the five-fold degeneracy, known as a projection on the laboratory axis of quantization, one can make any superpositions by using any wave functions that are associated with given level and in accordance with the general quantum-mechanical rule. The items of superposition must have the same row and column indexes up to sign, therefore every superposition have just four pieces. A rule used for obtaining superposition of rows must be the same as the rule which have been used by combining two columns.

$$
\begin{aligned}
& a_{1,1}+a_{1,-1}+a_{-1,1}+a_{-1,-1}=6 x_{1}^{2} x_{2}^{2}-6 x_{0}^{2} x_{3}^{2}+x_{0}^{4}-x_{1}^{4}-x_{2}^{4}+x_{3}^{4} \\
& a_{1,1}-a_{1,-1}-a_{-1,1}+a_{-1,-1}=6 x_{1}^{2} x_{3}^{2}-6 x_{0}^{2} x_{2}^{2}+x_{0}^{4}-x_{1}^{4}+x_{2}^{4}-x_{3}^{4} \\
& a_{2,2}-a_{2,-2}-a_{-2,2}+a_{-2,-2}=6 x_{2}^{2} x_{3}^{2}-6 x_{0}^{2} x_{1}^{2}+x_{0}^{4}+x_{1}^{4}-x_{2}^{4}-x_{3}^{4}
\end{aligned}
$$

Results of executing of operations mentioned above are listed in (A7). The terms on the right site are arranged up to factor of 2 and show a maximum similarity of the rotational wave functions for free molecules.

The final step from the rotational wave function for free molecules to those for latent molecules is obtained by using the Schmidt decomposition to lower the degree of terms in the right side of (A7). One can write the decomposition, for example, as $x_{1}^{2} x_{2}^{2}=\left(x_{1} x_{2}\right)\left(x_{1} x_{2}\right)$ for required elements, so we obtain the wave rotational functions for latent molecules with desired properties. The functions $x_{1} x_{2}, x_{1} x_{3}, x_{2} x_{3}$ are invariant under the spacial inversion therefore they represent simultaneously the direct and reverse rotations. Second, they have the appropriate symmetry properties listed in the last column of (A4) and they have the proper rotational energy that can be calculated using the angular operators of (A5) without terms involving variable $x_{0}$.

There is another approach to the problem of the rotational function of latent molecules based on the topological map or the Hopf map of three-dimensional sphere onto two-dimensional one. 
The starting point for the following considerations is the matrix of group SU(2) which matrix elements are complex combinations of coordinates in $R^{4}$.

$$
u=\left\|\begin{array}{rr}
z_{1} & z_{2} \\
-\bar{z}_{2} & \bar{z}_{1}
\end{array}\right\|=\left\|\begin{array}{cc}
x_{0}+i x_{1} & x_{2}+i x_{3} \\
-x_{2}+i x_{3} & x_{0}-i x_{1}
\end{array}\right\|
$$

There are three cases of the Hopf map $S^{3} \mapsto S^{2}$ depending on the quaternion chosen as imaginary unit [1]. The quaternion units are denoted with boldface in the following table.

$$
\left|\begin{array}{c|c|c}
u & z_{1} & z_{2} \\
x_{0} \mathbf{1}+x_{1} \mathbf{i}+\left(x_{2} \mathbf{1}+x_{3} \mathbf{i}\right) \mathbf{j} & x_{0}+i x_{1} & x_{2}+i x_{3} \\
x_{0} \mathbf{1}+x_{2} \mathbf{j}+\left(x_{3} \mathbf{1}+x_{1} \mathbf{j}\right) \mathbf{k} & x_{0}+i x_{2} & x_{3}+i x_{1} \\
x_{0} \mathbf{1}+x_{3} \mathbf{k}+\left(x_{1} \mathbf{1}+x_{2} \mathbf{k}\right) \mathbf{i} & x_{0}+i x_{3} & x_{1}+i x_{2}
\end{array}\right|
$$

The first step of Hopf map is a projection $S^{3} \mapsto C$ on extended complex plane (We include the case $z_{2}=0$ also).

$$
z=\frac{z_{1}}{z_{2}}
$$

The second step is conformal mapping $C \mapsto D$ of complex plane $C$ on unit disk $D$ (space compactification). As a rule this important step is not mentioned.

$$
w=\frac{2 z}{1+z \bar{z}}=2 z \bar{z} \quad z_{1} \bar{z}_{1}+z_{2} \bar{z}_{2}=1
$$

The coordinates on D are defined in the last step.

$$
x=(w+\bar{w}) / 2 \quad y=(w-\bar{w}) / 2 i \quad z^{2}=1-x^{2}-y^{2}
$$

The results of above operations are submitted in the following table. It is different from the conventional form of $\mathrm{SO}(3)$ matrix by row sequence in the first and second columns.

$$
\begin{aligned}
& \begin{array}{c|c|c|c|} 
& \mathbf{k} & \mathbf{j} & \mathbf{i} \\
x & 2 x_{0} x_{1}+2 x_{3} x_{2} & 2 x_{0} x_{3}+2 x_{2} x_{1} & 2 x_{0} x_{2}+2 x_{1} x_{3} \\
y & -2 x_{0} x_{2}+2 x_{3} x_{1} & -2 x_{0} x_{1}+2 x_{2} x_{3} & -2 x_{0} x_{3}+2 x_{1} x_{2} \\
z & 1-2 x_{1}^{2}-2 x_{2}^{2} & 1-2 x_{1}^{2}-2 x_{3}^{2} & 1-2 x_{2}^{2}-2 x_{3}^{2}
\end{array} \\
& S O(3)=\left\|\begin{array}{ccc}
x_{0}^{2}-x_{1}^{2}-x_{2}^{2}+x_{3}^{2} & -2 x_{0} x_{1}+2 x_{2} x_{3} & 2 x_{0} x_{2}+2 x_{1} x_{3} \\
2 x_{0} x_{1}+2 x_{2} x_{3} & x_{0}^{2}-x_{1}^{2}+x_{2}^{2}-x_{3}^{2} & -2 x_{0} x_{3}+2 x_{1} x_{2} \\
-2 x_{0} x_{2}+2 x_{1} x_{3} & 2 x_{0} x_{3}+2 x_{1} x_{2} & x_{0}^{2}+x_{1}^{2}-x_{2}^{2}-x_{3}^{2}
\end{array}\right\|
\end{aligned}
$$

The new point of this consideration is the relation between fundamental topology concept of Hopf map and $\mathrm{SO}(3)$ matrix structure.

\section{Appendix B}

A purpose of this Appendix is to give in the matrix form all spin states of water molecule including also mixed states to help for discussion which take place in Sec. 3.1.

Three possible arrangements of spin states for water molecules

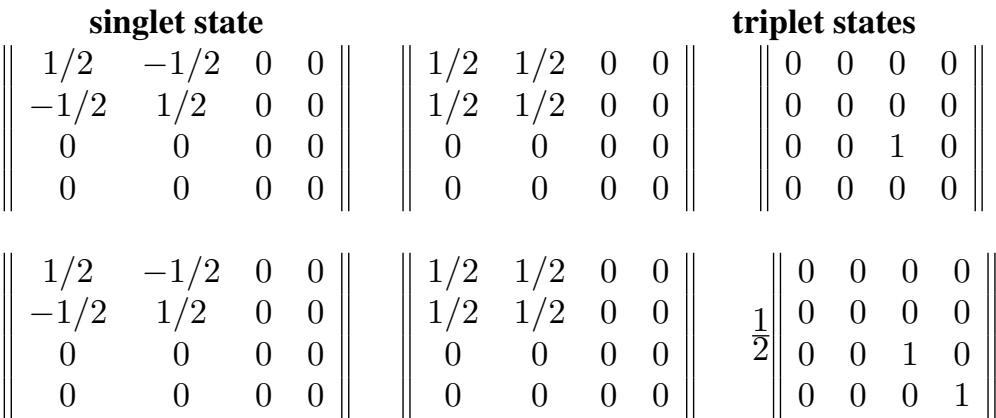

$$
\begin{aligned}
& \begin{array}{||cccc}
1 / 2 & -1 / 2 & 0 & 0 \\
-1 / 2 & 1 / 2 & 0 & 0 \\
0 & 0 & 0 & 0 \\
0 & 0 & 0 & 0
\end{array}\left\|\quad \begin{array}{l}
1 \\
3
\end{array}\right\| \begin{array}{cccc}
1 / 2 & 1 / 2 & 0 & 0 \\
1 / 2 & 1 / 2 & 0 & 0 \\
0 & 0 & 1 & 0 \\
0 & 0 & 0 & 1
\end{array} \|
\end{aligned}
$$

Spin phases for water molecules

\begin{tabular}{cc|cc} 
para- & \multicolumn{2}{c}{$\begin{array}{c}\text { ortho-molecules } \\
\text { triplet subspaces }\end{array}$} \\
phase & singlet subspace & \multicolumn{2}{|c}{ thet } \\
$\alpha$ & $\{|10\rangle-|01\rangle\}$ & $\{|10\rangle+|01\rangle\}$ & $\{|11\rangle\},\{|00\rangle\}$ \\
$\beta$ & $\{|10\rangle-|01\rangle\}$ & $\{|10\rangle+|01\rangle\}$ & $\{|11\rangle,|00\rangle\}$ \\
$\gamma$ & $\{|10\rangle-|01\rangle\}$ & & $\{|10\rangle+|01\rangle,|11\rangle,|00\rangle\}$
\end{tabular}


Here the brackets \{\} indicate the subspace in the Hilbert space associated with two spin-1/2 particles and the objects that are found within brackets are spin wave functions in conventional notations.

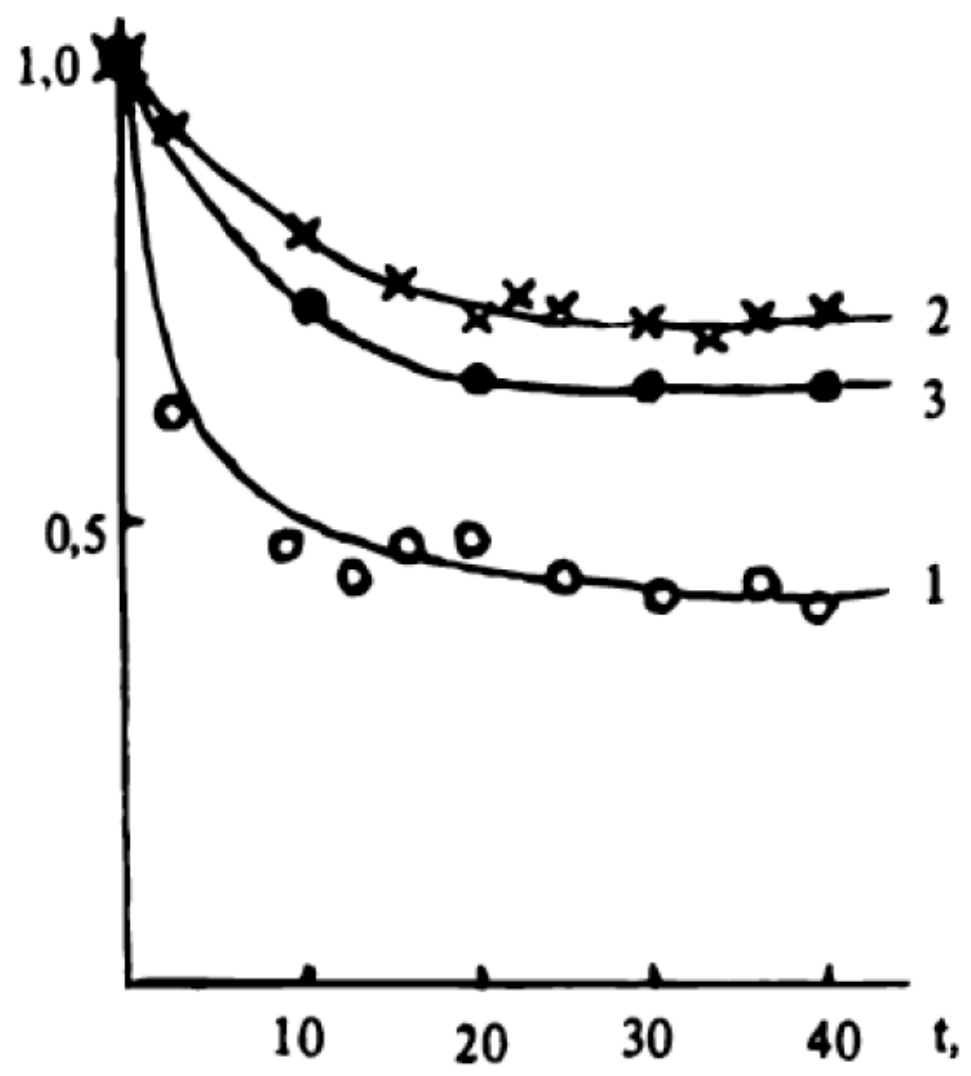

Figure 1. The concentration of ortho-molecules (curve 2), the concentration of para-molecules (curve 1) and the pressure in vacuum chamber (curve 3 ) as functions of time. All experimental points on Fig. 1 are normalized to the initial value of measured parameters. The time variable is measured in minutes, other parameters are expressed in relative units. 


\section{REFERENCES}

[1] F Berdon Alan. The geometry of discrete groups, 1983.

[2] Lawrence C Biedenharn, James D Louck, and Peter A Carruthers. Angular momentum in quantum physics: theory and application. Addison-Wesley Reading, MA, 1981.

[3] RF Curl Jr, Jerome VV Kasper, and Kenneth S Pitzer. Nuclear spin state equilibration through nonmagnetic collisions. The Journal of Chemical Physics, 46(8):3220-3228, 1967.

[4] Douglas Hugh Everett and Frank Sidney Stone. The structure and properties of porous materials, volume 10. Butterworths, 1958.

[5] VK Konyukhov. Dynamic model of ortho-para conversion of water molecules. Bulletin of the Lebedev Physics Institute, 38(3):76-79, 2011.

[6] VK Konyukhov. Spin states of para-water and orthowater molecule in gas and liquid phases. Physics and Chemistry of Liquids, 49(3):343-346, 2011.

[7] VK Konyukhov. The water molecule with conjoint translational, rotational and spin degrees of freedom in the gas and liquid phases. Universal Journal of Physics and Application, 2(6):302-309, 2014.

[8] VK Konyukhov, VI Tikhonov, and TL Tikhonova. Spin modification selective adsorption of water molecules on a surface of corundum ceramic. Kratk. Soobsh. Fiz. FIAN, (9):12-14, 1988.
[9] V.K. Konyukhov (ed.). Physical processes in lowtemperature gas-dynamic lasers. 1990.

[10] VK Konyukhov, VI Tikhonov, TL Tikhonova, and VN Faizulaev. Separation of spin-modifications of water and heavy water molecules. PIS'MA ZH. TEKH. FIZ,12(23):1438-1441, 1986.

[11] LevDavidovich Landau, Evgenij MLifsic, John Stewart Bell, and JB Sykes. Quantum mechanics: nonrelativistic theory, volume 3. Pergamon Press London, 1958.

[12] Andrea Miani and Jonathan Tennyson. Can ortho-para transitions for water be observed? The Journal of chemical physics, 120(6):2732-2739, 2004.

[13] JL Morrison and JF Hanlan. Solid/gas interface (proceedings of the second international congress of surface activity vol. ii), 1957.

[14] Kenneth SW Sing and SJ Gregg. Adsorption, surface area and porosity. Academic Press, London, pages 1-5, 1982.

[15] EV Stepanov, PV Zyryanov, and VA Milyaev. Laser analysis of the relative content of ortho-and para-water molecules for the diagnostics of spin-selective processes in gaseous media. Physics of Wave Phenomena, 18(1):33-43, 2010.

[16] VI Tikhonov, AM Makurenkov, VG Artemov, OE Porodinkov, and AA Volkov. Sorption experiments with water spin isomers in glycerol. Physics of Wave Phenomena, 15(2):106-110, 2007.

[17] N.Ya. Vilenkin. Special Functions and the Theory of Group Representations..., volume 22. AMS Bookstore, 1968. 